\title{
Prevenzione e terapia precoce del diabete mellito di tipo II: aspetti farmacoeconomici
}

\section{ANALISI ECONOMICA}

Lorenzo Pradelli*§

\begin{abstract}
Type II (non-insulin-dependent) diabetes is one of the most widespread chronic patologies in the developed countries and its prevalence in Italy is about $2-3 \%$ of the population. Type II diabetes is also associated with several other metabolic abnormalities such as central obesity, hypertension, and dyslipidemia, which contributes to the very high rate of cardiovascular morbidity and mortality. Therefore Type II diabetes involves a significant financial burden on the health care system.

The purpose of this paper is to explain the composition of the healthcare costs of managing people with Type II diabetes and the economic repercussions due to the adoption of an aggressive strategy against the pathology. To carry out this evaluation we considered the CODE-2 (The Cost of Diabetes in Europe - Type II) Study results, the American Diabetes Association Position Statement, the Diabetes Prevention Program and the UK Prospective Diabetes Study.

Evidence exists to show that introducing prevention program or an early therapy can avert or delay significantly the onset of cardiovascular morbidity in Type II diabetes patients. According to the pharmacoeconomical criteria, this very desiderable clinical goal is associated to a little increase of the health expenditures, and sometimes also to a costs saving.
\end{abstract}

Keywords: type II (non-insulin-dependent) diabetes, costs, prevention, early therapy Farmeconomia e percorsi terapeutici 2005; 6 (3): 251-261

\section{INTRODUZIONE}

Il diabete mellito non insulino dipendente (NIDDM), o di tipo II, è tra le patologie croniche più diffuse nei Paesi industrializzati, Italia compresa, con una prevalenza stimata nel nostro Paese intorno al 2-3\% della popolazione [1]. Vista la lunga durata della malattia e l'alta incidenza delle caratteristiche complicazioni micro- e macro-vascolari, non sorprende eccessivamente che la spesa sanitaria sostenuta per la cura di questi pazienti sia eclatante: è stato stimato che in Italia il costo sanitario diretto annuo ammonti a circa 5.200 milioni di euro, corrispondenti a quasi il $7 \%$ della spesa sanitaria (pubblica e privata) totale [2].

È ampiamente riconosciuto che la comparsa della grande maggioranza delle complicazioni del diabete (cardiovascolari, renali, metaboliche, ecc.) dipende strettamente dai livelli di zucchero nel sangue. L'adozione tem- pestiva, duratura e costante di misure efficaci di controllo glicemico già nelle fasi iniziali della malattia, addirittura prima che si giunga allo stadio di diabete conclamato, può prevenire o ritardare di anni le gravi malattie d'organo che accompagnano l'evoluzione del diabete.

In questo articolo verrà esaminata la formazione dei costi sanitari necessari a sostenere la gestione e la cura del paziente diabetico, così come le ricadute economico-sanitarie dei benefici clinici derivanti dall' adozione universale di una strategia aggressiva nella gestione del diabete mellito.

\section{LA FORMAZIONE DEL COSTO DI MALATTIA DEL DIABETE}

Lo studio CODE-2 (Cost of Diabetes in Europe-Type II Study) è stato progettato e condotto appositamente per rilevare il volume e le tipologie di risorse sanitarie assorbite dai 
pazienti diabetici con le attuali prassi cliniche in otto paesi europei (Italia, Germania, Francia, Inghilterra, Spagna, Belgio, Olanda e Svezia) [3]. La tecnica di rilevazione dei costi si è basata sulla somministrazione di due questionari, uno destinato ai medici di medicina generale (MMG) e uno rivolto ai pazienti.

Il questionario per il MMG, attraverso cui si sono ottenuti i dati clinici e quelli sull'utilizzo di risorse sanitarie dirette, indagava gli aspetti demografici, clinici ed economici, mentre il secondo, utilizzato per raccogliere informazioni di costo integrative, era orientato alla rilevazione del consumo di risorse dirette non sanitarie e indirette, della qualità di vita, della soddisfazione relativa al trattamento antidiabetico corrente e dell'utilizzo domiciliare dei test di autovalutazione delle concentrazioni di glucosio su sangue e urina. I principali risultati del CODE-2 sono riassunti in Tabella I.

Come si può osservare, $\mathrm{i}$ pazienti diabetici negli otto paesi dell' area euro che hanno partecipato allo studio CODE-2 sono più di 10 milioni, corrispondenti ad un $3 \%$ della popolazione totale, con un costo medio annuo per paziente di oltre 2.800 euro.

Di tali costi, la maggioranza viene sostenuta per le cure ospedaliere, seguite dalla cura ambulatoriale e infine dai farmaci. È da notare che la maggior parte dei costi farmaceutici non sono indotti dai trattamenti specifici per il diabete (ipoglicemizzanti orali e insulina), bensì da altre molecole, in particolare per i farmaci cardiovascolari. Già dall'esame superficiale di questi dati appare evidente come non sia tan- to la gestione routinaria, basata su visite e consulenza ambulatoriali e sulla somministrazione di antidiabetici orali e insulina, ad assorbire la maggior parte delle risorse investite nella cura del paziente diabetico, quanto piuttosto la prevenzione (farmaci cardiovascolari) e la cura (farmaci e ricoveri ospedalieri) delle complicanze vascolari. A conferma di ciò, vale la pena sottolineare che più della metà delle spese sanitarie è stata utilizzata per ricoveri ospedalieri, che si sono resi necessari "solo" nel 13\% dei pazienti arruolati nello studio CODE-2; viceversa, oltre il $60 \%$ dei diabetici era in trattamento con antidiabetici orali, il cui costo non ha però rappresentato più del $4 \%$ della spesa totale.

La presenza di complicazioni vascolari è effettivamente il maggiore cost-driver nella gestione del diabete: in Germania, ad esempio, il rapporto tra la spesa sanitaria sostenuta per il diabetico e quella di un paziente dalle medesime caratteristiche demografiche, ma senza diabete, passa da 1,3 per i pazienti senza complicazioni a 4,1 per i diabetici con complicazioni sia micro- che macro-vascolari [4]; in Spagna, il costo medio per paziente è stato di 833 euro in assenza di complicazioni, di 1403 in presenza di complicazioni microvascolari, di 2022 in presenza di quelle macrovascolari e, infine, di 2.133 euro nei pazienti con entrambi i tipi di complicazioni [5].

Nel complesso dei pazienti arruolati nel CODE-2, il $24 \%$ presentava entrambi i tipi di complicazione: il costo complessivo della loro gestione ha mostrato un aumento anche del $250 \%$ [6].
Tabella I

Principali risultati dello studio CODE-2 (modificata da [3])

\begin{tabular}{|c|c|c|c|c|c|c|c|c|c|}
\hline & Belgio & Francia & Germania & Italia & $\begin{array}{l}\text { Paesi } \\
\text { Bassi }\end{array}$ & Spagna & Svezia & $\begin{array}{l}\text { Regno } \\
\text { Unito }\end{array}$ & $\begin{array}{c}\text { Paesi } \\
\text { CODE-2 }\end{array}$ \\
\hline $\begin{array}{l}\text { Prevalenza NIDDM } \\
\text { (migliaia di soggetti) }\end{array}$ & 332 & 1.300 & 3.500 & 1.700 & 235 & 1.500 & 325 & 1.200 & $>10.000$ \\
\hline Prevalenza NIDDM (\%) & 3,3 & 2,2 & 4,2 & 3,0 & 1,7 & 3,9 & 3,6 & 2,0 & 3,0 \\
\hline Età media (anni) & 66,0 & 64,0 & 67,1 & 65,7 & 64,8 & 67,4 & 67,6 & 63,3 & 65,9 \\
\hline $\begin{array}{l}\text { Costi annuali totali } \\
\text { (milioni di euro) }\end{array}$ & 1.094 & 3.983 & 1.244 & 5.170 & 444 & 1.958 & 736 & 2.608 & 28.430 \\
\hline $\begin{array}{l}\text { \% della spesa } \\
\text { sanitaria complessiva }\end{array}$ & l & 3,4 & 6,5 & 6,6 & 1,6 & l & I & 2,5 & l \\
\hline $\begin{array}{l}\text { Costo medio/ } \\
\text { paziente/anno (euro) }\end{array}$ & 3.295 & 3.064 & 3.576 & 2.991 & 1.827 & 1.305 & 2.630 & 2.214 & 2.834 \\
\hline $\begin{array}{l}\text { Costi ospedalieri medi } \\
(\% \text { del totale) }\end{array}$ & 54 & 50 & 61 & 60 & 30 & 32 & 42 & 35 & 53 \\
\hline $\begin{array}{l}\text { Costi ambulatoriali } \\
\text { medi (\% del totale) }\end{array}$ & 18 & 22 & 11 & 19 & 25 & 26 & 31 & 38 & 24 \\
\hline $\begin{array}{l}\text { Costi farmaceutici } \\
\text { medi (\% del totale) }\end{array}$ & 27 & 27 & 28 & 22 & 46 & 43 & 27 & 26 & 23 \\
\hline $\begin{array}{l}\text { Costi antidiabetici orali } \\
\text { medi (\% del totale) }\end{array}$ & 4 & 7 & 3 & 2 & 6 & 5 & 1,5 & 3 & 4 \\
\hline $\begin{array}{l}\text { Costi antidiabetici orali } \\
\text { (\% della spesa } \\
\text { farmaceutica totale) }\end{array}$ & 14 & 25 & 12 & 10 & 12 & 11 & 6 & 10 & 13 \\
\hline
\end{tabular}


Le complicazioni, oltre che sui costi sanitari diretti, hanno un forte impatto anche sui costi intangibili, come emerge dalla valutazione della qualità di vita che è stata condotta contestualmente allo studio CODE-2. Lo strumento somministrato ai pazienti è l'EQ-5D, un questionario generico di valutazione della qualità di vita che esplora cinque dimensioni del benessere complessivo: mobilità, indipendenza, attività quotidiane, dolore e fastidio, oltre che la presenza di depressione e ansia. Tale questionario è stato completato da quasi 4.200 pazienti in 5 paesi, rivelando che lo stato di salute medio viene valutato tra buono e discreto $(0,69$ in una scala da $0=$ peggior stato di salute immaginabile a $1=$ stato di benessere totale) dal diabetico, con valori comunque inferiori a quelli dei coetanei senza diagnosi di diabete. Il punteggio ottenuto al questionario, come accennato, è risultato fortemente associato alla presenza e al tipo delle complicazioni diabetiche: i soggetti con diabete non complicato hanno totalizzato uno score medio di 0,76 , che si riduce a 0,69 in presenza di un solo tipo di complicazione, sia essa micro- o macrovascolare, fino a scendere ulteriormente fino al valore di 0,59 in caso di presenza concomitante di complicazioni di entrambi i tipi. [7]

Alcuni degli autori che hanno partecipato allo studio CODE-2 hanno anche analizzato in dettaglio, in lavori collegati, quanto del totale della spesa sia riconducibile, direttamente o indirettamente, al diabete e alle sue complicazioni. In Francia, ad esempio [8], è stato stimato che circa un quarto dei costi sanitari sia attribuibile direttamente al diabete, un altro quarto a possibili complicazioni e la restante metà sia invece da imputarsi ad altre patologie; in Spagna e in Italia la proporzione è risultata essere di circa un terzo per ciascuna delle tre voci $(29,30$ e $41 \%$ in Spagna e 29, 39 e $32 \%$ in Italia, rispettivamente) $[2,5]$. Il processo di imputazione dei costi utilizzato da questi autori si è basato sull'attribuzione alla diagnosi di diabete di quelle spese sostenute per la gestione o la cura del diabete stesso e delle condizioni che notoriamente lo accompagnano, come le complicazioni oculari, renali e cardiovascolari. Questa tecnica, però, probabilmente sottostima il reale onere del diabete, secondo i risultati di due ampi studi statunitensi condotti proprio con l'obiettivo di analizzare i costi incrementali indotti dalla diagnosi di diabete mellito non insulino dipendente, oltre che la loro evoluzione temporale. Per fare ciò, i ricercatori si sono avvalsi della disponibilità di un amplissimo database amministrativo e clinico, quello della Kaiser Permanente NorthWest Division (KPNW), una organizzazione privata di management sanitario, non-profit, operante nello stato dell'Oregon (Stati Uniti nordoccidentali), con circa 400.000 iscritti nel periodo in cui l'analisi è stata condotta. La tecnica utilizzata in questi due lavori $[9,10]$, una versione dello studio caso-controllo, è molto semplice e intuitiva, ma richiede appunto la disponibilità di dati completi relativi a una grande quantità di pazienti: per ogni soggetto con diagnosi di diabete presente nel database è stato selezionato un match, ossia un paziente dalle medesime caratteristiche demografiche e cliniche, fatta eccezione, ovviamente, della diagnosi di diabete, ed è stato confrontato il consumo di risorse sanitarie.

Il primo dei due lavori condotti con questo metodo di analisi ha analizzato la formazione e l'andamento dei costi incrementali legati alla diagnosi di diabete nei primi otto anni successivi alla data della diagnosi. Ai fini dell'analisi, sono stati inclusi i dati relativi a oltre 8500 diabetici (per quanto riguarda il primo anno dopo la diagnosi; a causa della tecnica utilizzata, il campione degli anni successivi è progressivamente minore); le tipologie di costi

\begin{tabular}{ccccc}
\hline \hline $\begin{array}{c}\text { Anni dalla } \\
\text { diagnosi }\end{array}$ & $\begin{array}{c}\text { Costi } \\
\text { controlli }\end{array}$ & $\begin{array}{c}\text { Costi } \\
\text { incrementali }\end{array}$ & $\begin{array}{c}\text { Costi } \\
\text { totali }\end{array}$ & $\begin{array}{c}\text { \% incremento/ } \\
\text { totale }\end{array}$ \\
\hline \hline 1 & $1.124 \pm 56$ & $1.243 \pm 106$ & $2.367 \pm 91$ & 53 \\
2 & $1.204 \pm 73$ & $791 \pm 115$ & $1.995 \pm 90$ & 40 \\
3 & $1.313 \pm 88$ & $668 \pm 132$ & $1.981 \pm 98$ & 34 \\
4 & $1.070 \pm 71$ & $954 \pm 135$ & $2.024 \pm 119$ & 47 \\
5 & $1.153 \pm 151$ & $1.012 \pm 201$ & $2.165 \pm 134$ & 47 \\
6 & $1.166 \pm 135$ & $853 \pm 196$ & $2.019 \pm 153$ & 42 \\
7 & $698 \pm 92$ & $1.340 \pm 182$ & $2.038 \pm 163$ & 66 \\
8 & $1.174 \pm 203$ & $1.375 \pm 368$ & $2.549 \pm 310$ & 54 \\
Totale & 8.902 & 8.236 & 17.138 & $/$ \\
Media annuale & $1.113 \pm 64$ & $1.030 \pm 93$ & $2.142 \pm 74$ & 48 \\
\hline \hline
\end{tabular}

Tabella II

Costi dei pazienti diabetici nei primi otto anni dalla diagnosi (modific ata da [9]) 
considerate comprendevano i ricoveri ospedalieri, le visite da medici generici e specialisti, i costi di farmaceutici e parafarmaceutici e le prestazioni ambulatoriali.

La diagnosi di diabete ha più che raddoppiato i costi sanitari rispetto a quelli sostenuti dai soggetti simili per età e sesso, ma senza diabete (Tabella II). Nei primi otto anni dalla diagnosi, ciò corrisponde a $2.633 \$$ (valuta del 1997) per anno e per paziente: l'incremento è immediatamente registrabile nel primo anno, per poi scendere lievemente nel secondo, prima di iniziare una salita costante nel rimanente periodo analizzato. Per ogni anno esaminato, la maggior parte dei costi incrementali è dovuta all'aumento delle spese ospedaliere (46\% dell'incremento complessivo), seguite dalle spese per farmaci e dispositivi medici e infine dai costi per le visite mediche [9].

Un dato molto interessante, cui si faceva riferimento in precedenza, è relativo alle cause

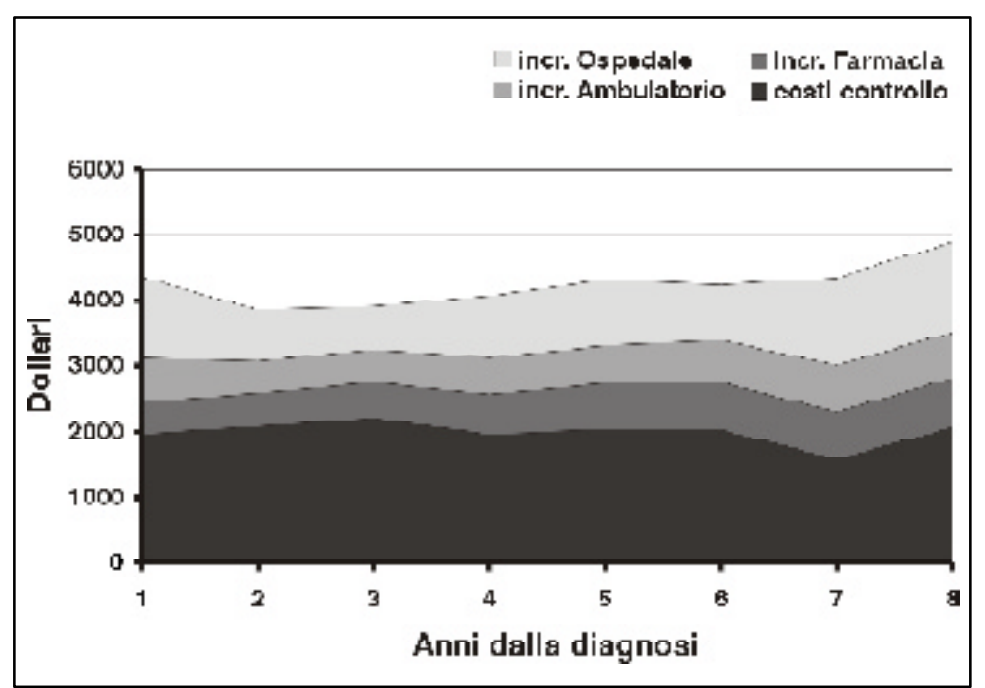

Figura 1

Formazione dell'eccesso di spesa nel diabetico (modificata da [9])

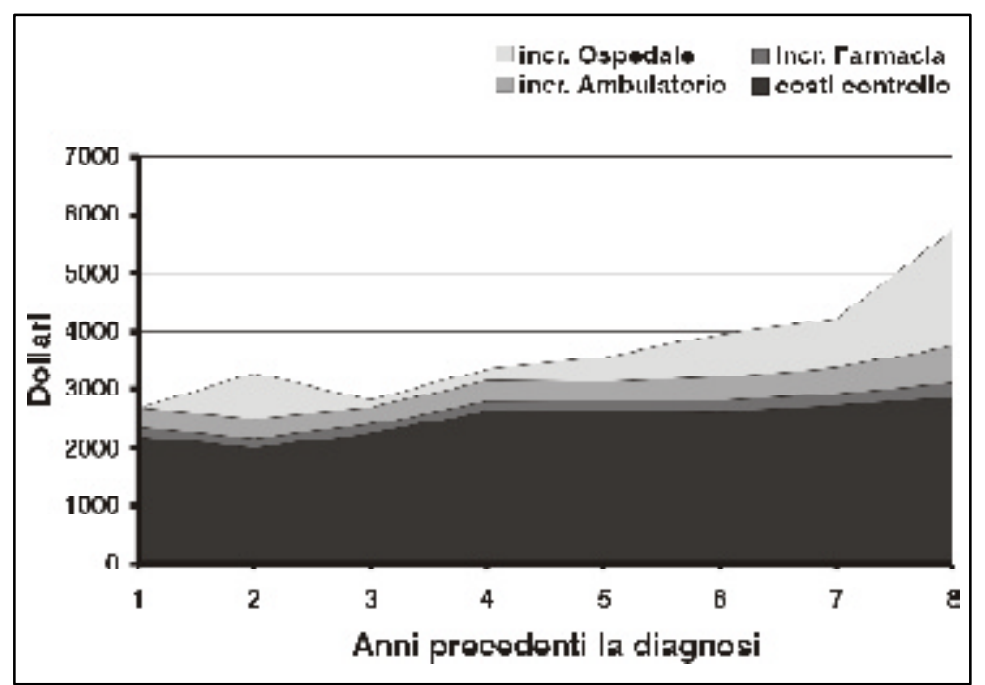

Figura 2

Formazione dell'eccesso di spesa nei pazienti con successiva diagnosi di diabete [10] dell'incremento dei costi nei diabetici: infatti, benché negli otto anni considerati la presenza di diabete abbia più che raddoppiato i costi per la gestione di cardiopatie, triplicato quelli per le patologie cerebrovascolari e quadruplicato i costi dei ricoveri per altre patologie cardiovascolari, ben il $64 \%$ dell' incremento di costo è stato speso per la cura di patologie che normalmente non verrebbero considerate come complicazioni del diabete. La Figura 1 mostra in dettaglio la formazione dell'eccesso di spesa indotto dalla diagnosi di diabete, evidenziando il ruolo delle varie componenti di costo incrementale.

Come si può osservare, $i$ costi dei pazienti controllo sono rimasti relativamente costanti nel corso del periodo di osservazione, nonostante gli otto anni di invecchiamento del campione. I costi incrementali del diabete, invece, dopo un picco di incremento registrato nel primo anno post-diagnosi, sono cresciuti linearmente a un ritmo approssimativo di 100 dollari all'anno. Anche in questo lavoro, il contributo della spesa per i farmaci e i dispositivi specifici contro il diabete (test di autovalutazione della glicemia) è relativamente basso, non superando il 5,9\% dei costi sanitari diretti totali.

Il medesimo gruppo di lavoro ha condotto un secondo studio utilizzando la medesima tecnica di caso-controllo, ma applicandola ai dati relativi agli otto anni precedenti la diagnosi di diabete, per verificare se l'eccesso di spesa fosse evidente già prima della data ufficiale di diagnosi (ricavata, in questo come nello studio precedente, o da una diagnosi ospedaliera o dalla prima prescrizione di un farmaco antidiabetico) [10].

Ai fini di quest'ulteriore analisi, sono stati analizzati i dati di oltre 9.500 diabetici (per l'ultimo anno precedente la diagnosi, progressivamente meno per quelli precedenti) e dei corrispettivi controlli. I costi sanitari necessari alla cura dei pazienti che successivamente avrebbero ricevuto la diagnosi di diabete sono stati significativamente superiori a quelli dei controlli per almeno 7 anni prima della data di prima diagnosi di diabete. Nell' arco degli otto anni precedenti questa diagnosi, il costo incrementale annuo medio dei pazienti con successiva evidenza di diabete è stato di 1.205 US\$ (valuta del 1998), con un' evidente accelerazione negli ultimi tre anni pre-diagnosi. Anche in questo caso, i costi ospedalieri sono responsabili della maggior parte $(53 \%)$ dell'eccesso di spesa. L'analisi condotta in questo lavoro si è ulteriormente estesa ai tre anni successivi alla diagnosi, confermando l'andamento osservato nel lavoro precedentemente descritto, con un balzo nel primo anno post-diagnosi, seguito da un aumento più graduale nel periodo successivo: ne risulta che il costo del primo anno post-diagnosi è quasi triplo rispetto a quello registrato solo due anni prima. Come 
nello studio precedente, in cui gran parte dell'incremento di costi sanitari non era dovuto a complicazioni o cure dirette del diabete, anche in questo lavoro l'aumento di spesa è dovuto in massima parte a condizioni cliniche apparentemente non collegate al diabete: il $78 \%$ dell'incremento dei costi ospedalieri è stato causato da patologie tradizionalmente non associate all'iperglicemia. Come osservano correttamente gli autori, tale dato sembrerebbe escludere la mancata diagnosi e cura del diabete tra i fattori rilevanti per l'aumento di costi osservato in questi pazienti.

La Figura 2 riassume la formazione dell'incremento di spesa nel periodo precedente la diagnosi di diabete.

\section{PREVENZIONE E TERAPIA DEL DIABETE MELLITO DI TIPO II: LINEE-GUIDA}

L'estrema rilevanza sociale, clinica ed economica del diabete dell' adulto ha condotto alla stesura, e costante revisione, di numerose linee guida sulla corretta gestione di questa patologia. In questa sede, ci interessa innanzitutto esporre quelle contenute nell'American Diabetes Association Position Statement [11], in quanto sono quelle che più specificatamente affrontano il tema della prevenzione, o perlomeno del rallentamento della progressione. Dopo una parte introduttiva in cui vengono illustrate le principali novità epidemiologiche e descritti gli studi clinici che hanno affrontato il tema, il documento viene svolto sotto forma di domanda e risposta. Riassumiamo i punti principali:

\section{1. È utile cercare di prevenire il diabete?}

Affinché abbia senso iniziare un programma di prevenzione su una patologia, devono essere soddisfatti almeno 5 requisiti:

a) la patologia in questione deve essere socialmente rilevante e comportare un significativo onere per chi ne è affetto.

Non vi è dubbio che il diabete soddisfi questo criterio;

b) lo sviluppo precoce e la storia naturale della patologia devono essere compresi quanto basta all'identificazione di parametri che ne indichino la progressione a patologia conclamata.

L'evidenza scientifica che indica come l'intolleranza al glucosio e l'iperglicemia a digiuno siano fortemente correlate allo sviluppo di diabete è molto robusta;

c) serve un test per l'identificazione dello stadio pre-clinico della patologia che sia sicuro, accettabile e predittivo.

Nel caso del diabete, due test soddisfano questi criteri: la glicemia a digiuno e il test da carico orale del glucosio; d) è necessario che ci sia almeno un tipo di intervento preventivo efficace, sicuro e affidabile.

Sulla base di una serie di recenti trial clinici, il gruppo di esperti dell'American Diabetes Association ha identificato almeno due interventi di questo tipo, ossia la modificazione dello stile di vita e la terapia con metformina. Inoltre, alcuni risultati promettenti sono stati ottenuti anche con altre classi di ipoglicemizzanti orali (tioglitazoni e inibitori dell'alfaglucosidasi);

e) lo sforzo necessario a individuare i soggetti a rischio e l'intervento preventivo non devono essere eccessivamente onerosi e devono possedere un buon rapporto di costo/efficacia.

Le dimostrazioni relative a quest'ultimo requisito sono ancora carenti, ma alcuni studi indicano che lo screening dei soggetti ad alto rischio di complicanze sia costo/efficace. Numerosi autori ritengono che per la popolazione generale, il metodo di screening più conveniente sia quello "opportunistico", ossia la rilevazione della glicemia a digiuno in occasione di contatti con il sistema sanitario avvenuti per altri motivi.

In conclusione, benché la costo-efficacia dello screening e degli interventi preventivi non sia definitivamente stabilita, l'elevato onere delle complicazioni diabetiche e i potenziali benefici aggiuntivi delle misure preventive (come la riduzione del rischio cardiovascolare e dell'obesità, per citare solo i più rilevanti) suggeriscono che valga la pena tentare di prevenire il diabete e le sue conseguenze.

\section{Quali soggetti vanno sottoposti a screening e ai trattamenti?}

Per rispondere a questa domanda, gli autori iniziano con la valutazione delle tecniche di screening disponibili, indicando che sia il test da carico che la glicemia a digiuno hanno le caratteristiche idonee per assolvere a questa funzione. In alternativa, è ipotizzabile l'utilizzo di modelli a punteggio, costruiti mediante l'esame di regressione logistica multifattoriale, che però mancano ancora del necessario processo di validazione. Partendo da queste e altre considerazioni, vengono fornite le seguenti raccomandazioni: è adeguato sottoporre a screening opportunistico i soggetti oltre i 45 anni, in particolare se il loro indice di massa corporea (BMI) è superiore a $25 \mathrm{~kg} / \mathrm{m} 2$. Sarebbe opportuno sottoporre a screening anche $\mathrm{i}$ soggetti di età inferiore, se presentano sovrappeso e almeno un altro fattore di rischio per lo sviluppo del diabete; il test andrebbe eseguito al mattino, in quanto più affidabile, $\mathrm{e}$ ripetuto ogni tre anni nei normoglicemici. 


\section{Come va eseguita la prevenzione del diabete?}

Il principale intervento che si è dimostrato in grado di prevenire la progressione degli stati iperglicemici a diabete conclamato è la modificazione dello stile di vita, inteso come riduzione del peso corporeo e aumento dell' attività fisica. L'altra strategia dimostratasi efficace è quella basata sull'utilizzo di agenti ipoglicemizzanti orali, particolarmente utile nei soggetti che non aderiscono ai programmi di terapia comportamentale. Nell'unico studio di confronto diretto (Diabetes Prevention Program, DPP), tuttavia, l'efficacia della terapia farmacologica (metformina) è risultata circa la metà di quella basata su riduzione del peso e aumento dell'esercizio. Solo nei soggetti relativamente giovani (24-44 anni) e obesi (BMI > $35 \mathrm{~kg} / \mathrm{m} 2)$ l'efficacia della metformina è risultata sovrapponibile a quella dell'intervento sullo stile di vita.

Per quanto riguarda il trattamento del diabete in fase clinica, riportiamo le principali raccomandazioni fornite nel 1999 dall'American College of Family Physicians [12] per la gestione e la prevenzione delle complicazioni diabetiche.

Secondo i medici di famiglia americani, va posta massima attenzione al trattamento dell'insulino-resistenza, per due ordini di ragioni fondamentali: l'associazione con altre condizioni patologiche, come intolleranza al glucosio, obesità centrale, dislipidemia, ipertensione e alterazioni della fibrinolisi, e la stretta correlazione esistente tra insulino-resistenza, iperglicemia e sviluppo di complicazioni vascolari. L'approccio all'insulino-resistenza è attuabile con metodi non farmacologici, cioè con la modificazione dello stile di vita, che rappresenta la prima scelta e va incoraggiata ad ogni contatto con il paziente, e con agenti farmacologici, ossia metformina e troglitazone (l'unico agente della classe dei tioglitazoni disponibile negli USA al momento della pubblicazione delle linee-guida e ritirato dal commercio nel 2000).

Per quanto riguarda le opzioni farmacologiche, riassumiamo di seguito le prese di posizione.

In generale, la terapia farmacologica orale è indicata nei pazienti diabetici in cui la dieta, l'esercizio e la riduzione ponderale sono risultati insufficienti al controllo glicemico.

Sulfoniluree: sono i primi ipoglicemizzanti orali introdotti in terapia e mantengono un ruolo di primo piano nella gestione del diabete; circa due terzi dei pazienti rispondono, benché sia necessario associare un secondo farmaco fino nel $20 \%$ dei casi; la sostituzione di una sulfonilurea con un' altra è utile in pochi casi; i prodotti dall'emivita più lunga sono sconsigliati negli anziani* e nei nefropatici, a causa dell' aumentato rischio di ipoglicemia; le sulfoniluree possono essere considerate farmaci di prima scelta nei pazienti non obesi.

Metformina: agisce riducendo l'insulinoresistenza e il rilascio epatico di glucosio; può essere somministrata in monoterapia o in combinazione; l'utilizzo in monoterapia non ha potenziale ipoglicemizzante, per cui sarebbe più corretto parlare di antiiperglicemizzante orale, piuttosto che di ipoglicemizzante; è controindicata nei pazienti con insufficienza renale e in caso di acidosi metabolica; presenta alcune interazioni farmacologiche da tenere presenti; è da considerarsi il farmaco di prima scelta come monoterapia iniziale nei pazienti obesi; è appropriato associare la metformina alla sulfonilurea nei pazienti non controllati in monoterapia; la metformina e i tioglitazoni sono le sole scelte logiche nei pazienti che necessitano di insulina, in quanto sono in grado di ridurre l'insulino-resistenza e di spezzare il circolo vizioso terapia insulinica / aumento ponderale / aumentata insulino-resistenza / perdita di controllo glicemico / aumento del fabbisogno insulinico.

Inibitori dell'alfa-glucosidasi: agiscono rallentando il catabolismo e l'assorbimento intestinale di monosaccaridi; sono indicati in monoterapia o in combinazione; possono costituire una valida alternativa alle sulfoniluree o alla metformina nei pazienti che non ottengono un controllo glicemico soddisfacente con questi farmaci e nei casi di insufficienza renale; il loro utilizzo è associato a epatotossicità che va monitorata; controindicati nei casi di malattia infiammatoria intestinale, ostruzione intestinale e altre patologie del tratta digerente.

Tioglitazoni (troglitazone)**: aumentano la sensibilità dei tessuti periferici all'azione insulinica e pertanto riducono l'insulino-resistenza; sono utili nei pazienti il cui diabete non risulta controllato nonostante alte dosi di insulina (permettono una riduzione del dosaggio insulinico fino al 50\%); ha potenziale epatotossico, per cui vanno monitorizzate le transaminasi fintanto che il paziente assume questi farmaci.

Repaglinide: farmaco dall'azione e dagli effetti collaterali simili a quelli delle sulfoniluree; costituisce una valida opzione nei pazienti intolleranti alle sulfoniluree; va utilizzata in monoterapia o in combinazione con la metformina; il dosaggio va titolato con cautela negli anziani e in caso di insufficienza epatica o renale.

Le sulfoniluree, la metformina e l'insulina si sono dimostrate in grado di prevenire lo sviluppo delle complicanze microvascolari in maniera simile e non è emersa differenza tra i loro outcomes cardiovascolari, per cui non vi è ragione clinica di preferire una classe rispetto all'altra come agente iniziale. 


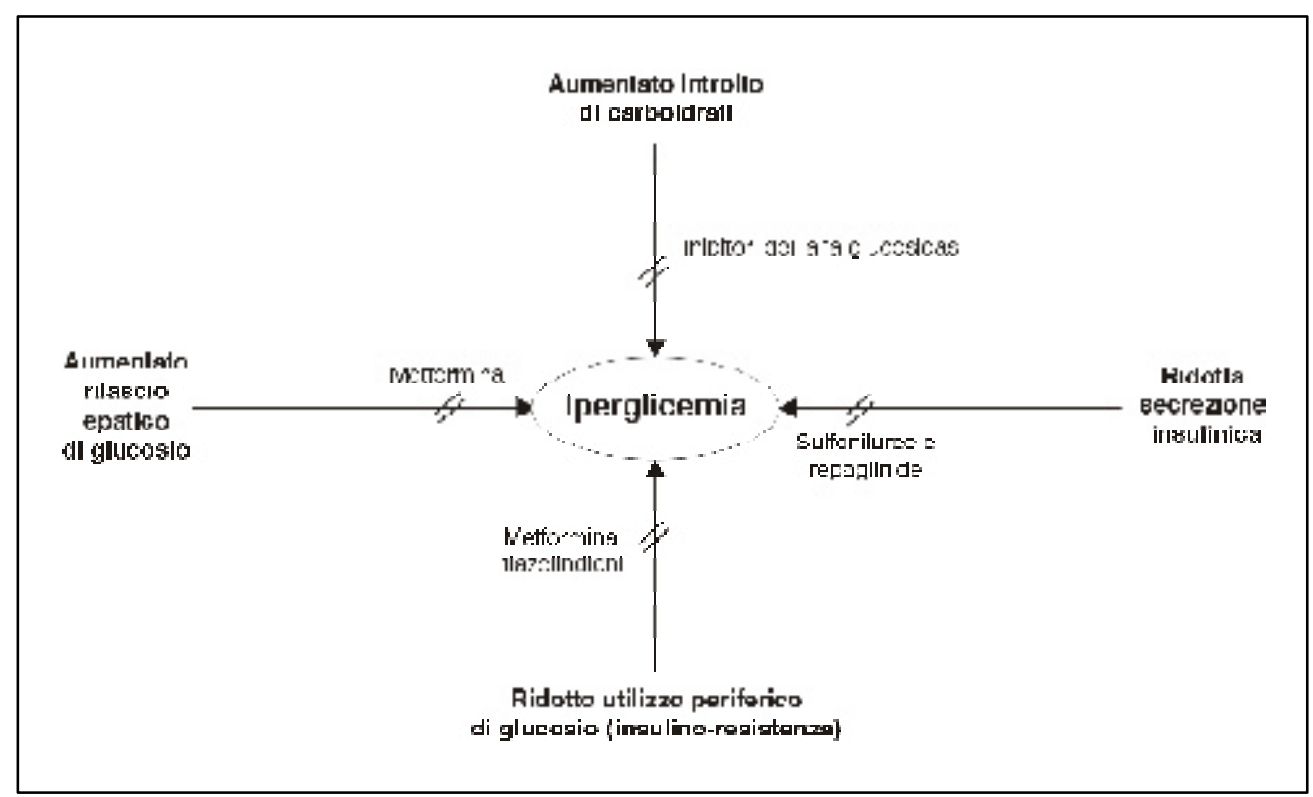

Combinazioni farmacologiche raccomandabili: sulfonilurea + metformina, sulfonilurea + inibitore alfa-glucosidasi, sulfonilurea + tioglitazone, repaglinide + metformina, tioglitazone + metformina, insulina + metformina e insulina + tioglitazone.

\section{EFFICACIA CLINICA E IMPATTO ECONOMICO NELLA GESTIONE DEL DIABETE}

La letteratura medica che ha affrontato il tema degli interventi terapeutici sull'intolleranza al glucosio e sul diabete è quasi sconfinata, per cui la descrizione dettagliata anche solo dei principali studi clinici non è possibile, né rientra negli scopi del presente lavoro. Abbiamo pertanto optato per la presentazione di una selezione di studi clinici, scelti sulla base della rilevanza e, soprattutto, della concomitante presenza di una valutazione economica dei risultati, per sottolineare come le terapie in grado di avere un impatto clinico rilevante sull'evoluzione della malattia siano generalmente caratterizzate da un ottimo profilo economico, a causa degli elevati costi delle complicazioni che si possono evitare. A giudicare dall'evidenza disponibile, appare che nella gestione del diabete le priorità cliniche coincidano quasi costantemente con gli interessi economici dei servizi sanitari, oltre che della società nel complesso.

\section{Diabetes Prevention Program (DPP) \\ - Prevenzione del diabete}

Lo studio DPP (Diabetes Prevention Program) [13] ha arruolato 3.234 pazienti di entrambi i sessi (donne $=68 \%$ ) con intolleranza al glucosio (IGT, Impaired Glucose Tolerance) di età superiore ai 25 anni (età media =51) e BMI almeno pari a $24 \mathrm{~kg} / \mathrm{m} 2$ (BMI medio $=34)$. I soggetti sono stati randomizzati in tre gruppi: al primo (gruppo terapia comportamentale) è stato impartito un ciclo di 16 lezioni iniziali, seguiti ogni mese da un counseling individuale e da uno di gruppo, per motivare i pazienti a raggiungere gli obiettivi prefissati, cioè una riduzione di almeno il $7 \%$ del peso corporeo di partenza mediante dieta e attività fisica moderata, da svolgersi per almeno 150 minuti/settimana; al secondo è stata somministrata metformina, alla dose di $850 \mathrm{mg}$ uid per un mese e successivamente di $850 \mathrm{mg}$ bid; il terzo gruppo ha ricevuto un placebo, somministrato con il medesimo schema posologico della metformina. Gli ultimi due gruppi hanno anch'essi ricevuto un counseling di terapia comportamentale, in forma scritta e attraverso una sessione annuale di 20-30 minuti in cui veniva enfatizzata l'importanza di una stile di vita salutare, oltre che una sessione quadrimestrale di incoraggiamento all'aderenza al trattamento con un case-manager.

Dopo un follow-up medio di 2,8 anni (range 1,8-4,6 anni), il gruppo di terapia comportamentale ha mostrato una riduzione del rischio di sviluppare diabete del 58\% (incidenza totale $4,8 \%$ ) e il gruppo trattato con metformina del $31 \%$ (incidenza totale $7,8 \%$ ), rispetto ai pazienti del gruppo placebo (incidenza del 11\%). In altri termini, sarebbe necessario includere 6,9 persone nel programma di intervento comportamentale, oppure somministrare metformina a 13,9 persone, per tre anni, per prevenire un nuovo caso di diabete. Il 50\% dei soggetti assegnati al gruppo di terapia comportamentale è riuscito a raggiungere l'obiettivo di calo ponderale prefissato (-7\%), e il $74 \%$ si è esercitato per almeno 150 minuti settimanali; nessun effetto indesiderato grave è stato osservato nei vari gruppi di trattamento. Nello studio di valutazione dell'impatto economico [14] delle due strategie preventive,
Figura 3

Cause di iperglicemia e siti d'azione dei farmaci antidiabetici orali (modificata da [12]) 
sono stati considerati i costi diretti e indiretti e adottate le prospettive del sistema sanitario e della società nel complesso. Poiché il protocollo sperimentale prevedeva una serie di analisi, di procedure e di interventi che difficilmente sarebbero realizzabili nella pratica clinica quotidiana, i ricercatori hanno anche analizzato i costi e i guadagni che si realizzerebbero applicando gli schemi terapeutici in ambito non sperimentale.

Per le valutazioni economiche sono state utilizzate due tecniche di analisi separate: è stata valutata la costo-efficacia, applicando i costi incrementali agli NNT (Number Needed to Treat, una misura di efficacia che esprime il numero di pazienti da trattare per un certo periodo necessario a evitare un evento), ottenendo il costo per caso di diabete evitato, e sono stati calcolati i rapporti di costo-utilità, ossia l'investimento economico necessario al guadagno di un QALY (anni di vita ponderati per la qualità). I principali risultati di queste analisi, così come delle relative analisi di sensibilità, sono riportate nelle tabelle III e IV.

Considerato l'elevato costo del diabete, un investimento di 15.000 o di 30.000 US\$, a se- conda che si attui la prevenzione con modificazioni dello stile di vita o con metformina, appare un prezzo relativamente contenuto per evitare o posticipare un nuovo caso della malattia. Inoltre, le analisi economiche condotte sui risultati di questo trial si sono limitate a valutare gli effetti economici prodotti nel corso della durata dello studio, senza prendere in considerazione l'evoluzione successiva della malattia: è altamente verosimile che la maggior parte dei benefici delle strategie preventive si esplichino nella loro interezza più avanti, per cui i rapporti di costo-efficacia calcolati in questo lavoro appaiono assai conservativi, a svantaggio degli interventi terapeutici. Vi è inoltre da considerare che i benefici clinici derivanti dalla modificazione dello stile di vita, e in misura minore anche dal trattamento dell'insulino-resistenza con metformina, non si riducono alla prevenzione del diabete, ma comprendono, ad esempio, la riduzione del rischio cardiovascolare e degli effetti deleteri dell'obesità [14]. In sintesi, i risultati della valutazione economica condotta sul DPP dimostrano che con l'adozione delle misure preventive basate su terapia comportamentale e
Tabella III

Costi per caso di diabete evitato:

scenario base e analisi di sensibilità (modificato da [14])

\begin{tabular}{|c|c|c|c|c|c|}
\hline \multicolumn{2}{|c|}{$\begin{array}{l}\text { Costo per caso di } \\
\text { diabete evitato(US\$) }\end{array}$} & \multicolumn{2}{|c|}{$\begin{array}{l}\text { Stile di vita } \\
\text { vs placebo }\end{array}$} & \multicolumn{2}{|c|}{$\begin{array}{l}\text { Metformina } \\
\text { vs placebo }\end{array}$} \\
\hline \multicolumn{2}{|l|}{ Prospettiva } & $\begin{array}{l}\text { Servizio } \\
\text { sanitario }\end{array}$ & Società & $\begin{array}{l}\text { Servizio } \\
\text { sanitario }\end{array}$ & Società \\
\hline \multicolumn{2}{|l|}{ Scenario base } & 15.655 & 24.426 & 31.338 & 34.489 \\
\hline \multirow{3}{*}{ Riduzione costi } & $25 \%$ & 11.755 & 20.526 & 24.606 & 27.757 \\
\hline & $50 \%$ & 7.855 & 16.626 & 17.874 & 21.025 \\
\hline & $75 \%$ & 3.956 & 17.727 & 11.141 & 14.292 \\
\hline \multirow{2}{*}{ Riduzione efficacia } & $10 \%$ & 17.243 & 26.904 & 34.406 & 37.865 \\
\hline & $20 \%$ & 18.831 & 29.382 & 37.693 & 41.483 \\
\hline Tasso di sconto & $3 \%$ & 15.804 & 24.581 & 31.452 & 34.604 \\
\hline
\end{tabular}

\begin{tabular}{cc|cc|cc}
\hline \hline \multicolumn{2}{c}{$\begin{array}{c}\text { Costo per QALY } \\
\text { guadagnato(US\$) }\end{array}$} & \multicolumn{2}{c}{$\begin{array}{c}\text { Stile di vita } \\
\text { vs placebo }\end{array}$} & \multicolumn{2}{c}{$\begin{array}{c}\text { Metformina } \\
\text { vs placebo }\end{array}$} \\
\hline Prospettiva & & $\begin{array}{c}\text { Servizio } \\
\text { sanitario }\end{array}$ & Società & $\begin{array}{c}\text { Servizio } \\
\text { sanitario }\end{array}$ & Società \\
\hline \hline Scenario base & 31.512 & 51.582 & 99.611 & 99.171 \\
& $25 \%$ & 23.662 & 43.732 & 78.212 & 77.772 \\
Riduzione costi & $50 \%$ & 15.811 & 35.880 & 56.814 & 56.373 \\
& $75 \%$ & 7.963 & 28.033 & 35.415 & 34.974 \\
& $10 \%$ & 35.013 & 57.313 & 11.679 & 110.190 \\
Riduzione efficacia & $20 \%$ & 39.389 & 64.477 & 124.514 & 123.964 \\
& & & & & 102.164 \\
\hline \hline
\end{tabular}

Tabella IV

Costi per QALY

guadagnato: scenario

base e analisi di

sensibilità (modificato da [14]) 


\begin{tabular}{lccc}
\hline \hline & $\begin{array}{c}\text { Strategia } \\
\text { intensiva }\end{array}$ & $\begin{array}{c}\text { Strategia } \\
\text { convenzionale }\end{array}$ & $\begin{array}{c}\text { Differenza } \\
\text { media }\end{array}$ \\
\hline \hline Costi medi per paziente (f del 1997) & & & \\
Terapia di base & 4.350 & 3.655 & 695 \\
Trattamento complicazioni & 5.258 & 6.215 & -957 \\
Totale trial & 9.608 & 9.869 & -261 \\
Totale trial scontato al 6\% & 6.958 & 7.170 & -212 \\
Totale in setting non sperimentale & 8.349 & 7.871 & 478 \\
Totale non sperimentale sconto 6\% & 6.027 & 5.689 & 338 \\
Anni medi di vita libera da eventi & & & \\
Within-trial & 14,89 & 14,29 & 0,60 \\
Within-trial scontati al 6\% & 9,17 & 8,88 & 0,29 \\
Stima che considera gli effetti post-trial & 15,08 & 13,94 & 1,14 \\
\hline \hline
\end{tabular}

metformina è possibile ottenere grandi vantaggi clinici a un prezzo decisamente accettabile per le economie dei paesi sviluppati.

\section{Studi UKPDS 41 e 34}

\section{- Controllo glicemico rigoroso}

Nello studio UKPDS 41, condotto dall'UK Prospective Diabetes Study group [15], sono stati arruolati 5.100 pazienti maggiori di 25 anni con nuova diagnosi di diabete di tipo II, definito come il riscontro di valori glicemici maggiori di $6 \mathrm{mmol} / \mathrm{L}$ in almeno due occasioni. Dopo una fase iniziale di terapia dietetica, 4.209 hanno raggiunto livelli glicemici a digiuno compresi tra 6 e $15 \mathrm{mmol} / \mathrm{L}$. Di questi, $342 \mathrm{sog}$ getti in sovrappeso sono stati assegnati a un trattamento con metformina, mentre i rimanenti sono stati randomizzati a una gestione tradizionale (1.138) basata fondamentalmente sulla dieta, al trattamento intensivo con insulina (1.156) o a uno con sulfoniluree (1.573). Il periodo di follow up è stato di 10 anni, gli outcomes clinici considerati sono stati la morte e le complicazioni diabetiche, tra cui cardiopatia coronarica, patologia cerebrovascolare, amputazioni, trattamento laser di retinopatia, rimozione di cataratta e insufficienza renale. Sono stati considerati solo i costi sanitari diretti e la prospettiva adottata è stata quella del servizio sanitario inglese.

Le strategie di controllo intenso della glicemia, nel loro insieme, hanno ridotto l'insorgenza di complicazioni diabetiche del $12 \%$ $(p=0,029)$, a fronte di un aumento medio del costo di trattamento di 659 US\$. Poiché non è risultata esservi alcuna differenza significativa in termini di mortalità, come outcome clinico ai fini dell' analisi economica è stato utilizzato il tempo libero da eventi patologici legati al diabete (infarto miocardico, scompenso cardiaco congestizio, ictus, dialisi, amputazione, estrazione di cataratta, emorragia vitrea e morte per qualsiasi causa), risultato in media di $15,08 \pm 7,2$ e di $13,94 \pm 6,89$ anni nel gruppo trattato e nel gruppo controllo, rispettivamente. Il rigoroso controllo della glicemia, inoltre, ha indotto una riduzione significativa dei costi delle complicazioni, di entità tale da compensare ampiamente l' aumento della spesa per farmaci: i costi totali medi sono stati o inferiori o non significativamente differenti da quelli della strategia convenzionale, a seconda che fosse applicato un tasso di sconto su costi e outcomes futuri o no. Non è dunque stato possibile né necessario calcolare un rapporto di costo/efficacia incrementale, in quanto le strategie più aggressive sono risultate in condizioni di dominanza, ossia al contempo più efficaci e meno costose. L'analisi economica è stata ripetuta sostituendo le prassi cliniche e i costi indotti dal protocollo sperimentale con parametri più vicini alla prassi clinica reale: in questo caso i costi totali dei pazienti assegnati alle strategie farmacologiche sono maggiori di quelli del gruppo in terapia dietetica, con un rapporto di costo efficacia incrementale di 563 \$/anno di vita senza eventi guadagnato.

Il gruppo UKPDS ha anche analizzato la convenienza a utilizzare metformina come terapia di prima linea nei soggetti sovrappeso con diabete di tipo II. A tale fine, 753 pazienti diabetici con peso corporeo superiore al $120 \%$ del peso ideale sono stati reclutati in un trial randomizzato e controllato vs la terapia convenzionale, prevalentemente di tipo dietetico [16]. Anche quest' analisi ha considerato unicamente i costi sanitari diretti e ha adottato la prospettiva del sistema sanitario britannico. Nel corso del trial (durata media 10,7 anni) la strategia di controllo stretto della glicemia con metformina ha indotto un aumento dell' aspettativa di vita di 0,4 anni, oltre a produrre un risparmio netto di risorse sanitarie (in media $258 £$ /paziente); è dunque risultata dominare l'alternativa ed è pertanto da considerasi la scelta più logica in questo tipo di pazienti.

\section{Tabella V}

Principali risultati clinici ed economici dell'UKPDS 41 (modificata da [15]) 


\section{CONCLUSIONI}

Il diabete mellito di tipo II, con la sua diffusione di tipo epidemico, comporta nei paesi industrializzati un onere economico notevolissimo, oltre a rappresentare una piaga sanitaria nelle nostre società.

Dal punto di vista economico, così come da quello clinico, le conseguenze più pesanti del diabete si manifestano nelle fasi avanzate della patologia, con la comparsa delle complicazioni micro- e macro-vascolari, principali responsabili della mortalità, del frequente ricorso alle strutture sanitarie e della perdita di qualità di vita legate a questa patologia.
L'evidenza scientifica indica che l'identificazione e la terapia precoce dei soggetti con condizioni pre-diabetiche, così come le strategie terapeutiche mirate a mantenere un controllo stretto dei valori glicemici nei pazienti in cui il diabete è già manifesto, sono in grado di prevenire o di procrastinare in maniera significativa la comparsa del danno vascolare. Gli studi di economia sanitaria condotti in merito hanno dimostrato, inoltre, che questo obiettivo altamente desiderabile dal punto di vista clinico è raggiungibile con un aumento modesto della spesa sanitaria, se non addirittura con un concomitante risparmio di costi.

\section{BIBLIOGRAFIA}

1 Nicolucci A, Scorpiglione N, Belfiglio M, Carinci F, Cavaliere D, el-Shazly M, Labbrozzi D, Mari E, Massi Benedetti M, Tognoni G . Patterns of care an Italian diabetic population. The Italian Study Group for the Implementation of the St Vincent Declaration, Società Italiana di Diabetologia, Associazione Medici Diabetologi. Diabet Med. 1997 Feb;14(2):158-66

2 Lucioni C, Garancini MP, Massi-Benedetti M, Mazzi S, Serra G per conto dell'Advisory Board Italiano dello studio CODE-2 - Il costo sociale del diabete di tipo 2 in Italia: lo studio CODE-2. PharmacoEconomics, Italian Research Articles, 2000;1(2):1-21

3 Jonsson B; CODE-2 Advisory Board. Revealing the cost of type II diabetes in Europe. Diabetologia. 2002 Jul;45(7):S5-12

4 Liebl A, Spannheimer A, Reitberger U, Gortz A. Costs of long-term complications in type 2 diabetes patients in Germany. Results of the CODE-2 Study Med Klin (Munich). 2002 Dec 15;97(12):713-9

5 Mata M, Antonanzas F, Tafalla M, Sanz P. The cost of type 2 diabetes in Spain: the CODE-2 study. Gac Sanit. 2002 Nov-Dec;16(6):511-20

6 Williams R, Van Gaal L, Lucioni C; CODE-2 Advisory Board. Assessing the impact of complications on the costs of Type II diabetes. Diabetologia. 2002 Jul;45(7):S13-7

7 Koopmanschap M; CODE-2 Coping with Type II diabetes: the patient's perspective. Diabetologia. 2002 Jul;45(7):S18-22

8 Detournay B, Fagnani F, Pribil C, Eschwege E. Medical resources consumption of type 2 diabetics in France in 1998 Diabetes Metab. 2000 May;26(3):225-31

9 Brown JB, Nichols GA, Glauber HS, Bakst AW. Type 2 diabetes: incremental medical care costs during the first 8 years after diagnosis. Diabetes Care. 1999 Jul;22(7):1116-24

10 Nichols GA, Glauber HS, Brown JB. Type 2 diabetes: incremental medical care costs during the 8 years preceding diagnosis. Diabetes Care. 2000 Nov;23(11):1654-9

11 American Diabetes Association Position Statement. Prevention or delay of type 2 diabetes. Diabetes Care 2004 Jan, Suppl.1:S47-54 
12 Florence JA et Yeager BF. Treatment of Type 2 Diabetes Mellitus. American Family Physician 1999 May; 59(10). Disponible on-line all'indirizzo http://www.aafp.org/afp/990515ap/2835.html

13 Molitch ME, Fujimoto W, Hamman RF, Knowler WC; Diabetes Prevention Program Research Group. The diabetes prevention program and its global implications. J Am Soc Nephrol. 2003 Jul;14(7 Suppl 2):S103-7

14 Diabetes Prevention Program Research Group. Within-trial cost-effectiveness of lifestyle intervention or metformin for the primary prevention of type 2 diabetes. Diabetes Care. 2003 Sep;26(9):2518-23

15 Gray A, Raikou M, McGuire A, Fenn P, Stevens R, Cull C, Stratton I, Adler A, Holman R, Turner R. Cost effectiveness of an intensive blood glucose control policy in patients with type 2 diabetes: economic analysis alongside randomised controlled trial (UKPDS 41). United Kingdom Prospective Diabetes Study Group. BMJ. 2000 May 20;320(7246):1373-8

16 UK Prospective Diabetes Study (UKPDS) Group. Effect of intensive blood-glucose control with metformin on complications in overweight patients with type 2 diabetes (UKPDS 34). Lancet. 1998 Sep 12;352(9131):854-65

17 Rosenkranz B, Profozic V. et al. Pharmacokinetics and safety of glimepiride at clinically effective doses in diabetic patients with renal impairment. Diabetologia, 1996; 39(12):1617-24

18 Massi-Benedetti M. Glimepiride in type 2 diabetes mellitus: a review of worldwide therapeutic experience. Clin Ther, 2003; 25(3):799-816

19 Proprietà farmacocinetiche, RCP, giugno 2004 
